\title{
Journal of Nephropathology
}

\section{Mycophenolate mofetil versus cyclophosphamide for idiopathic membranous nephropathy; a double blind and randomized clinical trial}

CrossMark

\author{
Fatemeh Hayati $^{1}{ }^{\circledR}$, Heshmatollah Shahbazian ${ }^{1}$, Ali Ghorbani ${ }^{1}$, Shahla Ahmadi Halili ${ }^{*}{ }^{* \oplus}$, Eisa Rezaei ${ }^{1}$, \\ Leila Sabetnia ${ }^{1}$, Seyed Seifollah Beladi Mousavi ${ }^{1,2}$
}

${ }^{1}$ Chronic Renal Failure Research Center, Ahvaz Jundishapur University of Medical Sciences, Ahvaz, Iran

${ }^{2}$ Tehran University of Medical Sciences, Baharloo Hospital, Tehran, Iran

\begin{tabular}{|c|c|}
\hline ARTICLE INFO & ABSTRACT \\
\hline Article type: & \multirow{14}{*}{$\begin{array}{l}\text { Introduction: The current treatment regimens for patients with idiopathic membranous nephropathy } \\
\text { (MN) are based on cyclophosphamide-glucocorticoid or calcineurin inhibitor-glucocorticoid. } \\
\text { Objectives: We evaluated whether mycophenolate mofetil (MMF) -glucocorticoid could be an option } \\
\text { for first-line therapy among these patients. } \\
\text { Patients and Methods: In a double-blinded, randomized and controlled clinical trial, we compared } \\
\text { the effect of MMF with cyclophosphamide in inducing complete or partial remission (PR) among } \\
\text { patients with nephrotic syndrome due to idiopathic MN. All of the patients in both groups also } \\
\text { received steroid, renin-angiotensin blockers and statins. Diuretics were also used in the patients who } \\
\text { had edema. The primary end point of our study was change in urinary protein/creatinine ratio. } \\
\text { Results: A total of } 30 \text { patients completed the study. Around } 17 \text { patients received MMF ( } 2 \mathrm{~g} / \mathrm{d}) \text { and } 13 \\
\text { patients received intravenous or oral cyclophosphamide for } 6 \text { months. At the start of the study, no } \\
\text { significant differences in demographic and biochemical parameters of patients including the urinary } \\
\text { protein excretion rate between two groups }(P=0.432) \text {. The proportion of proteinuria was } 5235 \pm 1655 \\
\text { mg/24 in MMF group and } 8781 \pm 8741 \mathrm{mg} / 24 \text { in the cyclophosphamide group at the beginning of } \\
\text { the study. The rate of complete and PR were } 5.9 \% \text { and } 52.9 \text { in MMF group versus } 16.7 \% \text { and } 100 \% \\
\text { in cyclophosphamide group which it is significantly lower in MMF group. Kidney function was } \\
\text { stable in both groups during treatment. } \\
\text { Conclusions: According to the result of our study, a } 6 \text {-month therapy with MMF-glucocorticoid is not } \\
\text { recommended for treatment of patients with nephrotic syndrome due to idiopathic MN. }\end{array}$} \\
\hline Original & \\
\hline Article & \\
\hline $\operatorname{Rec}$ & \\
\hline & \\
\hline & \\
\hline & \\
\hline & \\
\hline My & \\
\hline Nephrotic syndrome & \\
\hline & \\
\hline & \\
\hline & \\
\hline & \\
\hline
\end{tabular}

Implication for health policy/practice/research/medical education:

Membranous nephropathy (MN) is among the most common causes of the nephrotic syndrome in adults who do not have diabetes mellitus. It can be caused by a variety of underlying diseases, infections and a variety of drugs like penicillamine or bucillamine. However MN is idiopathic in approximately $75 \%$ of cases. The treatment of MN among patients who have secondary form of $\mathrm{MN}$ is an effective treatment of the underlying disease. In contrast to secondary form of $\mathrm{MN}$, in primary $\mathrm{MN}$, immunosuppressive regimens including cyclophosphamideglucocorticoid or calcineurin inhibitor-glucocorticoid are administered. In this study, we evaluated whether mycophenolate mofetilglucocorticoid could be an option among these patients.

Please cite this paper as: Hayati F, Shahbazian H, Ghorbani A, Ahmadi Halili S, Rezaei E, Sabetnia L, et al. Mycophenolate mofetil versus cyclophosphamide for idiopathic membranous nephropathy; a double blind and randomized clinical trial. J Nephropathol. 2019;8(2):e12. DOI: $10.15171 /$ jnp.2019.12.

\section{Introduction}

Membranous nephropathy $(\mathrm{MN})$ is among the most common causes of the nephrotic syndrome in adults who do not have diabetes mellitus (1).

While the relative frequency of MN on kidney biopsy has declined in recent years compared to focal segmental glomerulosclerosis, however, it is still accounting for up to one-third of biopsy diagnoses in adults (particularly over age 40 years) with the nephrotic syndrome in some regions $(1,2)$.

MN can be caused by a variety of underlying diseases and infections including systemic lupus erythematosus, 
sarcoidosis, malignancy, and hepatitis $\mathrm{B}$ and $\mathrm{C}$ virus infection. In addition, it can also be caused by a variety of drugs like penicillamine, bucillamine, gold salts, antiTNF therapy and nonsteroidal anti-inflammatory drugs (NSAIDs). However MN is idiopathic in approximately $75 \%$ of cases (3-8).

The treatment of $\mathrm{MN}$ among patients who have secondary form of $\mathrm{MN}$ is effective treatment of the underlying disease, eradication of infections and or cessation of the offending drug which are usually associated with improvement in the MN $(2,3)$.

In contrast to secondary form of $\mathrm{MN}$, immunosuppressive agents should be considered among some patients who have an idiopathic form of MN (9).

However clinical course of idiopathic $\mathrm{MN}$ is benign in a significant percent of patients and spontaneous complete and partial remission (PR) of proteinuria at five years occur in $5 \%$ to $30 \%$ and $25 \%$ to $40 \%$ respectively $(9,10)$.

Therefore according to the potential toxicity of immunosuppressive agents, it is suggested that these drugs should be considered only in those patients who are most at risk for progressive disease (11).

The most important predictors of risk for a progressive disease among patients who have an idiopathic form of $\mathrm{MN}$ are persistent severe proteinuria particularly if protein excretion exceeds 8 to $10 \mathrm{~g} / \mathrm{d}$ and a reduced creatinine clearance at presentation or over the assessed proteinuria period $(11,12)$. Untreated these patients are most at risk for progression to end-stage renal disease which is a life-threatening disease with significant health consequences and poor outcomes (13-17). The primary immunosuppressive regimens used to treat idiopathic MN include cyclophosphamide -glucocorticoid and calcineurin inhibitor-glucocorticoid (9-11).

\section{Objectives}

In this study, we evaluated whether mycophenolate mofetil (MMF) -glucocorticoid regimen could be an option among these patients.

\section{Patients and Methods \\ 3.1. Study design}

The study was a double-blind, randomized and controlled clinical trial approved by the Research Center of Ahvaz Jundishapur University of Medical Sciences. Our study performed at the outpatient clinic of nephrology in the Golestan hospital, Ahvaz, Iran. The drugs (MMF and cyclophosphamide) were provided to the patients free of cost. The period of study was twelve months from July 2014 to January 2018. Before beginning of the trial, the nature of the study was explained to each patient by author and written informed consents were obtained from the participants. The primary end-point of the study was reduction of proteinuria in both MMF and the cyclophosphamide groups. The study design is shown in Figure 1.

\subsection{Inclusion, exclusion and randomization}

Adult patients, who referred to our clinic with nephrotic

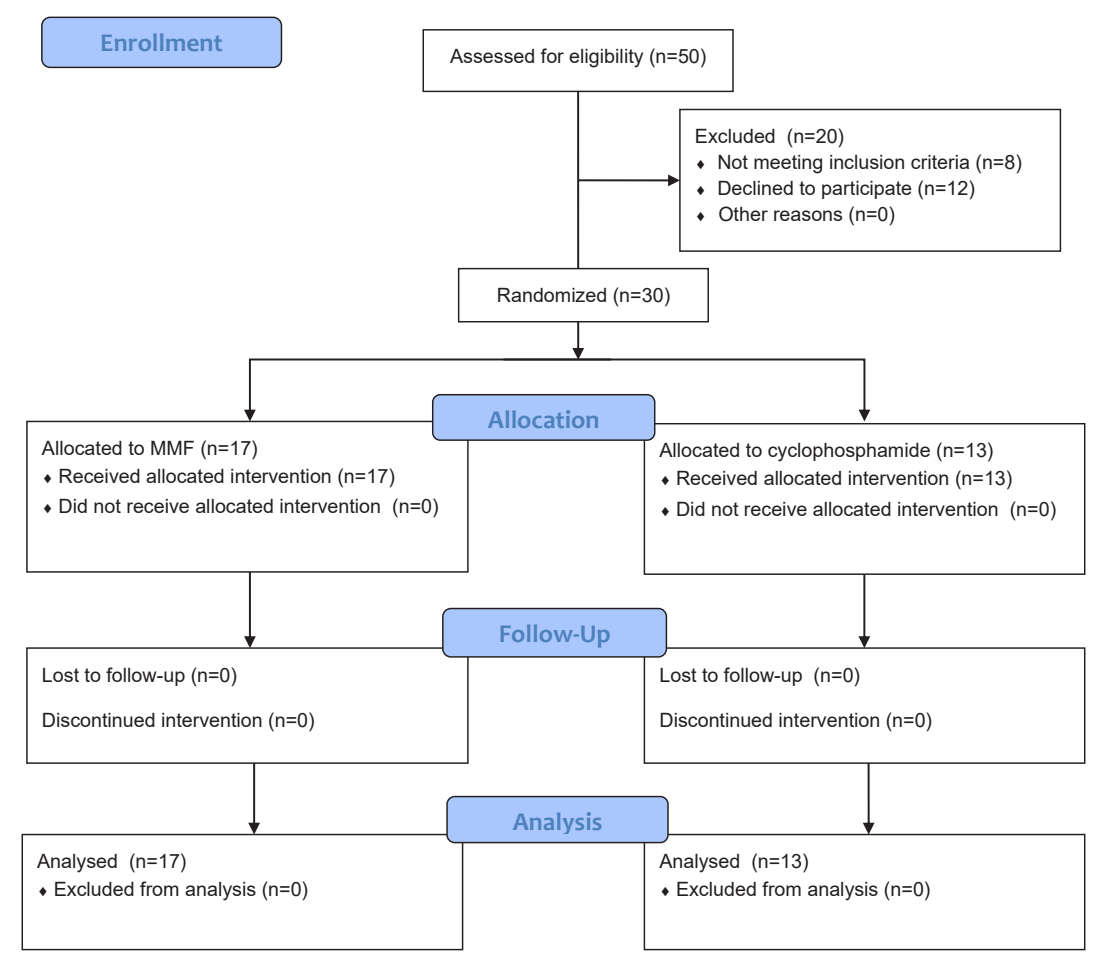

Figure 1. CONSORT (consolidated standards of reporting trial) chart for the study. 
syndrome and biopsy-proven MN by light microscopy and immunofluorescence, were evaluated. Nephrotic syndrome was defined as proteinuria more than 3 to $3.5 \mathrm{~g} / \mathrm{d}$ along with hypoalbuminemia and hyperlipidemia.

We used a standardized questionnaire to collect general information of our patients including age, gender, vital signs, body mass index (BMI), the record of previous diseases, type and dose of immunosuppressive and immunosuppressive medications such as angiotensinconverting enzyme inhibitors (ACEI) and angiotensin II receptors blockers (ARB), and the results of laboratory data.

The inclusion criteria for selection of patients were patients with nephrotic syndrome and biopsy-proven $\mathrm{MN}$ who were older than 18 years, patients who had persistent proteinuria despite six months of non-immunosuppressive medications with ACEI or ARB and patients who had creatinine clearance more than $60 \mathrm{~mL} / \mathrm{min}$.

MN patients who had massive proteinuria (more than 8 $\mathrm{g} / \mathrm{d}$ ) and MN patients who had creatinine clearance less than $60 \mathrm{~mL} / \mathrm{min}$ were also included and immunosuppressive therapy was initiated among these patients at the same time as ACEI or ARB initiation.

MN patients with the following characteristics were excluded from the study; loss of follow up, patients who had systemic diseases including diabetes mellitus, hepatitis B or $\mathrm{C}$ virus positivity, active infection, malignancy, renal vein thrombosis and acute coronary syndrome, pregnant women and patients who had active peptic ulcer disease and could not tolerate immunosuppressive therapy.

We randomly allocated our patients in two groups (MMF and cyclophosphamide). Patients in the MMF group received MMF at $2 \mathrm{~g} / \mathrm{d}$ in 2 divided doses for 6 months. They also received prednisolone at $0.5 \mathrm{mg} / \mathrm{kg} / \mathrm{d}$ for 2 to 3 months. The dose of MMF was decreased to 1.5 or 1 $\mathrm{g} / \mathrm{d}$ in three or two divided doses among patients who had gastrointestinal symptoms with MMF.

Patients in the MMF group received a course of alternate months of steroid in the first, third, and fifth months and cyclophosphamide at 1.5 to $2 \mathrm{mg} / \mathrm{kg} / \mathrm{d}$ in the second, fourth, and sixth months. The steroid months were began with pulse methylprednisolone, $1 \mathrm{~g}$ intravenously daily for 3 consecutive days, without oral prednisone and then followed by oral prednisolone at $0.5 \mathrm{mg} / \mathrm{kg} / \mathrm{d}$ for 27 days.

In both groups, hypertension was treated with dietary salt restrictions, ACE inhibitors and ARB. Among hypertensive patients, additional antihypertensive agents including calcium channel blockers and diuretics were added to achieve appropriate blood pressure control.

Dietary restrictions, statins and/or fibric acid derivatives were used among patients who had hyperlipidemia.

Patients in two groups were followed monthly and or more frequently when required during treatment for evaluation of therapy, side effects of medications and blood pressure monitoring. Laboratory parameters including urinalysis, 24- hour urinary protein excretion rate, complete blood count, serum creatinine, blood urea nitrogen (BUN), serum albumin, fasting blood sugar, cholesterol and triglyceride were monitored at each visit while MMF and cyclophosphamide were discontinued temporarily when the white blood cell count fell to less than $4000 \mu \mathrm{L}$ and or platelets decreased to less than $100000 \mu \mathrm{L}$.

Complete remission (CR) was defined to exist when the protein excretion rate was below $300 \mathrm{mg} / \mathrm{d}$ together with normal renal function on at least three occasions and PR was defined when the protein excretion level was below 3.5 $\mathrm{g} / \mathrm{d}$ plus a $50 \%$ or greater reduction in protein excretion from previous values together with normal renal function.

\subsection{Ethical issues}

The research followed the tenets of the Declaration of Helsinki. Informed consents were obtained from all patients. The study was approved by the ethical committee of Ahvaz Jundishapur University of Medical Sciences (ethical code; IR.AJUMS.REC.1393.226). This paper is a part of nephrology fellowship thesis of Shahla Ahmadi Halili, in the department of nephrology of Ahvaz Jundishapur University of Medical Sciences. Besides that, the study protocol was registered as in the Iranian registry of clinical trials (identifier: IRCT20180128038539N1; http://irct.ir/ trial/29433).

\subsection{Statistical analysis}

In order to compare the averages, the ratios and variables classified among groups were used in the statistical tests ( $t$ test, Mann-Whitney, chi-square and Fisher exact test). Also in order to investigate the duration of the relative and complete remission in patients, Kaplan-Meier survival duration curve was used. To compare the survival curves in two groups log-rank test was used. The Cox regression model was used to introduce the risk factors affecting the patient's recovery. In the end, all analyses were carried out using SPSS 19 and $P$ value $\leq 0.05$ was significant.

\section{Results}

A total of 30 patients with nephrotic syndrome and biopsyproven MN (19 males and 11 females) met eligibility criteria and completed the study. They were randomly assigned to the MMF group (17 patients; 10 males and 7 females) and the cyclophosphamide group (13 patients; 9 males and 4 females). The mean age of patients in MMF and the cyclophosphamide group $s$ were $38.11 \pm 7.27$ and $38.69 \pm 6.52$ years with no significant difference between them $(P=0.15)$.

There was also no significant difference between females and males in both MMF and the cyclophosphamide group s $(P=0.6$; Figure 2$)$.

In addition, at the start of the study, no significant differences in demographic and biochemical parameters of patients including the urinary protein excretion rate between two groups was detected $(P=0.432)$. 


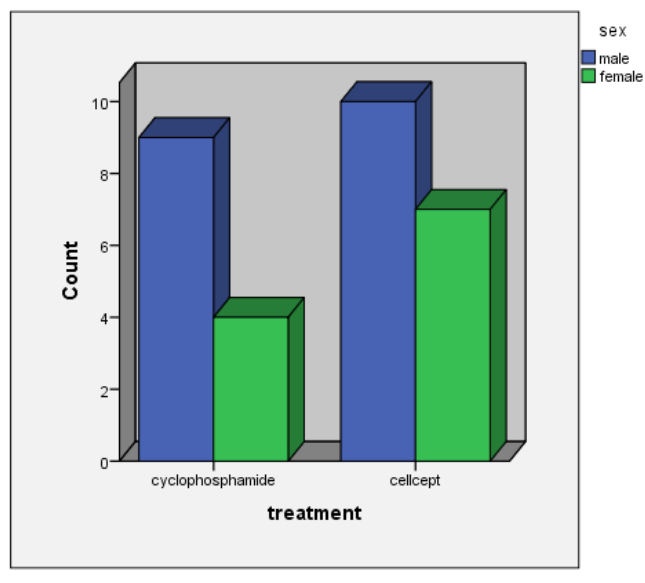

Figure 2. Females and males in both groups.

The proportion of proteinuria was $5235 \pm 1655$ $\mathrm{mg} / \mathrm{d}$ in the MMF group and $8781 \pm 8741 \mathrm{mg} / \mathrm{d}$ in the cyclophosphamide group at the beginning of the study $(P=0.432)$.

At the end of the sixth month of therapy, all of the patients in the cyclophosphamide group (100\%) and 52.9\% in the MMF group achieved complete or partial remission which is significantly lower in the MMF group.

Complete remission occurred in two male patients in the clophosphamide group (16.7\%) and one female patients in the MMF group (5.9\%).

Partial remission occurred in 11 patients (83.3\%) (7 males and 4 females) in the cyclophosphamide group and 8 patients (47.05) (5 males and 3 females) in the MMF group.

Eight patients (47.05) (5 males and 3 females) in the MMF group did not achieve complete or PR after course of therapy.

In summary, the rate of complete and PR were $5.9 \%$ and 52.9 in the MMF group versus $16.7 \%$ and $83.3 \%$ in the cyclophosphamide group which is significantly better in the cyclophosphamide group.

Kidney function was stable in both groups. Accordingly, creatinine clearance did not differ significantly either within or between groups during treatment.

\section{Discussion}

MMF, a powerful inhibitor of lymphocyte proliferation, has been administered since the early 1990s for the treatment of transplant patients. More recently, it has also been tried for treatment of patients with a variety of autoimmune diseases including diffuse or focal proliferative lupus nephritis and a variety of primary glomerular diseases including idiopathic MN (18-23).

Several prospective trials have suggested that MMF can be used as initial therapy in the great majority of patients with diffuse or focal proliferative lupus nephritis (class III or IV) and it has an effect at least equivalent (but not superior to) to cyclophosphamide among these patients (19).
Various clinical trials have also demonstrated that an MMF induction regimen provides similar efficacy and possibly fewer serious adverse effects compared with cyclophosphamide among patients with lupus nephritis $(19,20)$.

There are a number of investigations about the effect of MMF in the treatment of idiopathic MN, both as first-line therapy like our study and also in patients who have failed prior therapy and the results are conflicting (21-23).

Our study compared the effect of MMF-glucocorticoid with cyclophosphamide-glucocorticoid in inducing complete or PR among patients with idiopathic MN and demonstrated that the rate of complete and PR in the MMF group is significantly lower than the MMF group .

At the end of the sixth month of therapy, all of our patients in the MMF group achieved complete or PR which is significantly better than the MMF group. The rate of complete and PR in the MMF group was only about $60 \%$. Therefore according to the result of our study, we could not recommend MMF-glucocorticoid as an as initial therapy among patients with idiopathic MN.

Similar to the results of our study, Dussol et al showed that MMF is not effective for first-line therapy among these patients. In the randomized trial of Dussol et al, 36 patients with idiopathic $\mathrm{MN}$ who had protein excretion ranging from 5 to $10 \mathrm{~g} / \mathrm{d}$ investigated. All patients received conservative therapy and 19 patients received MMF at 2.0 $\mathrm{g} / \mathrm{d}$ in addition to conservative therapy. After 12 months follow up, the rates of CR and PR were not any significant differences between the two groups (21).

In contrast to the results of Dussol et al and our study, there are some randomized trials and observational studies which have suggested that MMF may be as effective as cytotoxic agents for first-line therapy among patients with idiopathic MN (22,23).

As an example, Senthil Nayagam et al showed that a 6-month therapy with MMF at $2.0 \mathrm{~g} / \mathrm{d}$ along with prednisolone at $0.5 \mathrm{mg} / \mathrm{kg} / \mathrm{d}$ for $2-3$ months is as effective as conventional protocol (monthly cycles of steroids and cyclophosphamide for 6 months) for primary treatment of idiopathic MN (22).

Similar findings were noted in randomized study of Chan et al which compared the effect of MMF- prednisolone with chlorambucil- prednisolone among 20 patients with MN who were most at risk for progressive disease. According to the results of this investigation, MMF- prednisolone provided similar efficacy compared with chlorambucilprednisolones and there was no significant difference between two groups in the rate of complete and PR (23).

\section{Conclusions}

$\mathrm{MN}$ is among the most common causes of the nephrotic syndrome in adults who don't have diabetes mellitus. It can be caused by a variety of underlying diseases, infections and drugs. However MN is idiopathic in approximately $75 \%$ 
of cases.

In contrast to secondary form of $\mathrm{MN}$, immunosuppressive agents should be considered among some patients who have idiopathic form of MN.

The current primary regimens administered to treat these patients include alkylating agents (cyclophosphamide or, less often, chlorambucil) with steroids or calcineurin inhibitor drugs (cyclosporine or tacrolimus) with or without steroids.

We compared the effect of MMF-glucocorticoid with cyclophosphamide-glucocorticoid in inducing complete or $\mathrm{PR}$ among patients with idiopathic $\mathrm{MN}$ and demonstrated that the rate of complete and PR in the MMF group is significantly lower than the MMF group. In contrast to the results of our study, other randomized trials have suggested that MMF may be as effective as cytotoxic agents for firstline therapy among idiopathic MN patients. Therefore further randomized controlled clinical trials are needed to ascertain the efficacy of MMF among patients with an idiopathic form of $\mathrm{MN}$.

\section{Study limitations}

Our investigation is limited by the short duration and the small number of Patients enrolled in the study. In addition, our investigation was also a single-center clinical trial. Therefore multi-center studies with larger number of patients and longer duration are needed to further evaluate the effect of MMF-glucocorticoid in treatment of these patients. While few clinical trials have suggested that MMF can be administered as a first line therapy among patients with idiopathic MN. However the beneficial effect of MMF was not detected in various randomized trials including our study. Therefore further long-term data and randomized controlled clinical trials are needed to prove the effect of MMF among these patients.

\section{Authors' contribution}

HS and FH designed the study, observed accuracy and validity of the study. LA collected the data and follow the study. HS, SS, SA and IR supervised the project. LA and A-GH wrote the paper. All authors edited and revised the final manuscript and accepted its publication.

\section{Conflicts of interest}

The authors declared no competing interests.

\section{Ethical considerations}

Ethical issues (including plagiarism, misconduct, data fabrication, falsification, double publication or submission, redundancy) have been completely observed by the authors.

\section{Funding/Support}

We declare no source of funding on this project.

\section{References}

1. Glassock RJ. The treatment of idiopathic membranous nephropathy: A dilemma or a conundrum? Am J Kidney Dis. 2004; 44:562-6.

2. Cattran D. Predicting outcome in the idiopathic glomerulopathies. J Nephrol 1998;11:57-60.

3. Haas M, Meehan SM, Karrison TG, Spargo BH. Changing etiologies of unexplained adult nephrotic syndrome: a comparison of renal biopsy findings from 1976-1979 and 1995-1997. Am J Kidney Dis. 1997;30:621-31.

4. Braden GL, Mulhern JG, O'Shea MH, Nash SV, Ucci AA $\mathrm{Jr}$, Germain MJ. Changing incidence of glomerular diseases in adults. Am J Kidney Dis. 2000; 35(5):878-83.

5. Korbet SM, Genchi RM, Borok RZ, Schwartz MM. The racial prevalence of glomerular lesions in nephrotic adults. Am J Kidney Dis. 1996;27:647-51.

6. Wasserstein AG. Membranous glomerulonephritis. J Am Soc Nephrol 1997;8:664.

7. Nagahama K, Matsushita H, Hara M, Ubara Y, Hara S, Yamada A. Bucillamine induces membranous glomerulonephritis. Am J Kidney Dis. 2002;39(4):706-12.

8. Stokes MB, Foster K, Markowitz GS, Ebrahimi F, Hines W, Kaufman D, et al. Development of glomerulonephritis during anti-TNF-alpha therapy for rheumatoid arthritis. Nephrol Dial Transplant. 2005;20(7):1400-6.

9. Ponticelli C, Passerini P. Other immunosuppressive agents for focal segmental glomerulosclerosis. Semin Nephrol. 2003;23:242-8.

10. Ponticelli C, Zucchelli P, Passerini P, Cagnoli L, Cesana B, Pozzi C, et al. A randomized trial of methylprednisolone and chlorambucil in idiopathic membranous nephropathy. N Engl J Med. 1989;320:8-13.

11. Shiiki H, Saito T, Nishitani Y, Mitarai T, Yorioka N, Yoshimura A, et al. Prognosis and risk factors for idiopathic membranous nephropathy with nephrotic syndrome in Japan. Kidney Int. 2004;65(4):1400-7.

12. Troyanov S, Roasio L, Pandes M, Herzenberg AM, Cattran DC. Renal pathology in idiopathic membranous nephropathy: a new perspective. Kidney Int. 2006;69(9):1641-8.

13. Beladi Mousavi SS, Alemzadeh Ansari MJ, Cheraghian B. Outcome of patients on hemodialysis in Khuzestan, Iran. NDT Plus. 2011;4(2):143-4.

14. Beladi Mousavi SS, Tavazoe M, Hayati F, Sametzadeh M. Arterio-venous fistula recirculation in hemodialysis: causes and prevalences. Shiraz E Med J. 2010;11(4):219-24.

15. Zeraati A, Beladi Mousavi SS, Beladi MM. A review article: access recirculation among end stage renal disease patients undergoing maintenance hemodialysis. Nephrourol Mon. 2013;5(2):728-32. doi: 10.5812/numonthly.6689.

16. Beladi-Mousavi SS, Motemednia F, Beladi Mousav M. Epidemiology of hepatitis $\mathrm{E}$ virus infection in patients on chronic hemodialysis. Jundishapur J Microbiol. 2014;7(5):e6993

17. Beladi Mousavi SS, Hayati F, Valavi E, Rekabi F, Beladi Mousavi M. Comparison of survival in patients with endstage renal disease receiving hemodialysis versus peritoneal dialysis. Saudi J Kidney Dis Transpl. 2015;26(2):392-7.

18. Fulton B, Markham A. Mycophenolate mofetil. A review of its pharmacodynamic and pharmacokinetic properties and clinical efficacy in renal transplantation. Drugs. 1996; 
51:278.

19. Appel GB, Contreras G, Dooley MA, Ginzler EM, Isenberg D, Jayne D, et al. Mycophenolate mofetil versus cyclophosphamide for induction treatment of lupus nephritis. J Am Soc Nephrol. 2009;20(5):1103-12.

20. Chan TM, Li FK, Tang CS, Wong RW, Fang GX, Ji $\mathrm{YL}$, et al. Efficacy of mycophenolate mofetil in patients with diffuse proliferative lupus nephritis. Hong KongGuangzhou Nephrology Study Group. N Engl J Med. 2000;343(19):1156-62.

21. Dussol B, Morange S, Burtey S, Indreies M, Cassuto E, Mourad G, et al. Mycophenolate mofetil monotherapy in membranous nephropathy: a 1-year randomized controlled trial. Am J Kidney Dis. 2008;52(4):699-705.

22. Senthil Nayagam L, Ganguli A, Rathi M, Kohli HS, Gupta KL, Joshi K, et al. Mycophenolate mofetil or standard therapy for membranous nephropathy and focal segmental glomerulosclerosis: a pilot study. Nephrol Dial Transplant. 2008;23(6):1926-30.

23. Chan TM, Lin AW, Tang SC, Qian JQ, Lam MF, Ho YW, et al. Prospective controlled study on mycophenolate mofetil and prednisolone in the treatment of membranous nephropathy with nephrotic syndrome. Nephrology (Carlton). 2007;12(6):576-81.

Copyright (C) 2019 The Author(s); Published by Society of Diabetic Nephropathy Prevention. This is an open-access article distributed under the terms of the Creative Commons Attribution License (http://creativecommons.org/licenses/by/4.0), which permits unrestricted use, distribution, and reproduction in any medium, provided the original work is properly cited. 